\title{
A New Methodology for Designing a Skull Implant
}

\author{
Nassim Markiz ${ }^{1^{*}}$, Eszter Horváth ${ }^{1}$ and Péter Ficzere ${ }^{1}$ \\ ${ }^{1}$ Department Vehicle Elements and Vehicle Structure, Faculty of Transport and Vehicle Engineering, \\ Budapest University of Technology and Economics, Budapest, Müegyetem Rkp. 3, 1111, Hungary.
}

\section{Authors' contributions}

All authors contributed to the study conception and design. Material preparation, data collection and analysis were performed by authors NM, EH and PF. The first draft of the manuscript was written by author NM and all authors commented on previous versions of the manuscript. All authors read and approved the final manuscript.

Article Information

DOI: 10.9734/ARRB/2021/v36i930420

(1) Dr Paola Angelini, University of Perugia, Italy:
Reviewers:

(1) Sonali Bhutad, Mumbai University, India. (2) Mgr. Volodymyr Polishchuk, Uzhhorod National University, Ukraine. Complete Peer review History: https://www.sdiarticle4.com/review-history/68649

Original Research Article

Received 09 April 2021

Accepted 15 June 2021

Published 03 September 2021

\begin{abstract}
Cranioplasty is a surgery used to repair a bone defect in the skull caused by an injury. It involves lifting the scalp and restoring the contour of the skull with an implant usually manufactured by additive manufacturing. The cranial implant is a sensitive topic; thus, it must be manufactured to the highest standards. Medical implants are growing significantly due to industrial digitalization and the rapid development of industrial software. With the help of computed Tomography (CT) equipment, a spatial, rotating model of the patient's current state can be obtained quickly, even in minutes where the replacement part of the deficiency can be perfectly designed. Although this requires considerable routine, computational capacity, and time, but taking advantage of the latest software presented in our manuscript, the development time of the implant can be up to 50 times shorter with significant improvements in suitability and adaptability. Subsequently, we can produce more accurate implants with more accessible and faster manufacturing with our developed method. The development steps and methods of designing an implant are described in our article.
\end{abstract}

Keywords: Skull; computed tomography; cranioplasty; computer-aided design; patients. 


\section{INTRODUCTION}

Head injury is a typical term used to describe any trauma to the head, and most precisely to the brain itself. It contributes dramatically to the outcome in one-half of all deaths from traumas. The inquiry generally results from motor vehicle accidents, jumping from high places such as trees. Moreover, children also have head injuries due to violence and abuse $[1,2]$. The skull is a protective cover of the brain, the sense organs, and the basis of the face [3]. A cranial bone defect is often caused by traumatic bone destruction, where the scalp, the skull, or the brain can also be damaged. In other cases, a cranial tumor, congenital disabilities result in functional and aesthetic deficiencies. However, there are solutions with the design and manufacturing of standardized implant templates (SDT), but the repair of severe skull injuries can be done using customized cranial implants $[2,4]$. The application of medical image analysis has constantly been growing with the availability of several open-source image processing and visualization software. However, the use of this software in the clinical environment has been limited because additional work is usually necessary, or a good job needs a lot of time, and sometimes the problem requires a unique solution. Medical doctors are generally using this visualization software only as a viewer to diagnose the problem. Therefore, they do not know to edit the possibilities. On the other hand, this requires the intervention of engineers to work with this software without knowing the medical background. In many cases, these works require strong cooperation between medical doctors and engineers [5].

Craniofacial reconstruction is a highly complex surgery because the operated body parts contain the brain, eyes, and other sensory organs. Sometimes a bone defect has a particular shape with different curvatures in various planes, so the implant has to be individually adjusted by a neurosurgeon during the surgery, which increases blood loss, infection risk, and the operating time [6]. The best way of treating cranial defects would be autogenous bone transplantation because this will have fewer complications of infections when compared to implants from other materials [7]. Currently, titanium is the commonly used material for cranial reconstruction due to its excellent biocompatibility, customization, and mechanical performance. However, several non-carcinogenic biocompatible materials are lighter such as polymethylmethacrylate

Polyetheretherketone

(PEEK),

(PMMA), and

hydroxyapatite (HA), which would be better as an implant. Still, each has its shortcomings, such as the risk of infections and lesser strength [8]. A computer numerically controlled (CNC) milling machine can also be used to manufacture a mold from which an implant could be cast [9]. Cranial implants do not require extra strength, so plastic materials are usable for this point of view. Furthermore, it has lower thermal conductivity, which is very important because, this way, the brain is not exposed to extreme temperatures so easily.

Furthermore, magnetic resonance imaging examinations induce electrical currents in these medical implants. These eddy currents may heat the implants and thus may be capable of causing localized tissue heating. Some implants can be made from transparent material, which is an additional advantage of plastics. This way, the organ can be seen without severe operation. In our case, ultra-high molecule-weight polyethylene (UHMW-PE) was chosen as implant material. This material is a very rigid, noncorrosive, nontoxic thermoplastic. It is relatively cheap and has good radiological transparency [10]. In general, standard implants are not suitable in these surgeries of skull defects compared to joint prostheses because of the complexity and the difference in the anatomy of the skulls. Research on skull prosthesis modeling can be found in the literature [45,9,11-13]. Therefore, the cranial implant is fabricated on a customized and individual basis based on case differences. This study aims to introduce a new methodology to design a patientspecific implant (PSI), a uniquely shaped implant for every unique patient based on different medical imaging data such as CT or Cone Beam Computer Tomography (CBCT).

\section{MATERIALS AND METHODS}

\subsection{Image Processing}

Image Processing Toolbox (IPT) of MATLAB provides a wide range of advanced image processing functions for enhancing and analyzing digital images. Spatial image transformations, morphological operations such as edge detection and noise removal, region-ofinterest processing, filtering, curve fitting are available using IPT functions, which are implemented in the open MATLAB language can be used to develop the customized algorithms. 


\subsection{CT scan defect}

The input information for three-dimensional (3D) modeling is given in our case by a CT scan. The objective of this research is to reconstruct the damaged skull. The digital imaging and communications in medicine (DICOM) files made by CT equipment of the examined bone were used to construct a 3D model. These files can be displayed as grayscale layers, where the brightness of each pixel corresponds to the absorption of X-rays in a thin rectangular layer of the cross-section, which is called a "voxel." These files contain other data that refer to the patient, the distance of the layers, and the size of the objects on the pictures. Latter geometrical information is vital for image processing. Most tissue types represent different weakening ranges [14]. Thus, after converting DICOM files to a picture file format using image processing methods, we separate the examined tissue, in this case, the bone from its environment and create a model [15]. The image files from the CT recordings were processed using MATLAB, one of the most versatile software in the engineering field. Our program can automatically load and process the image layers following code without any action. It is possible to run the program automatically because the images are derived from one record and their sizes are the same in pixels and the distance of each pixel in the specified directions is the same. The name of the images has been also sorted and the distance between the layers is the same. This means that all the program needs are to start processing a new $\mathrm{CT}$ record using the input parameters listed above.

The program reads and processes the image file one by one in the proper order. After reading, each image is stored as a 2-dimensional $(512 * 512)$ matrix, where each element of the matrix corresponds to one pixel. From here, the processing operations use these matrices. The first step in processing is masking: removing parts with greater intensity around the skull. (Fig. 1) shows a polygon defined around the damaged skull, which forms a mask, and the intensity of the pixels outside the mask is reduced. Segmentation of anatomical images is a general but essential step in medical imaging processing. It assigns the same label to every pixel in the content-related region (e.g., brain, bone, or scalp). Bone can usually be segmented with simple algorithms such as thresholding owing to the apparent difference in the Hounsfield scale in comparison to the surrounding tissues [16]. This was also the next step in our case; the image dataset was binarized, whereby the 2dimensional matrix will contain only ones and zeroes, where the threshold is set according to the intensity limit for each pixel. However, other techniques were also applied to finish the segmentation of CT data due to inhomogeneous bone structure and inherent blurring. The bone was closed, and tiny spots appeared right after binarization was removed. After performing series of morphological operations to form the skeleton of the feature of interest (i.e., region of the broken skull) or the whole skull, a 3D matrix $\left(512^{*} 512^{*} 170\right)$ containing 170 layers is stored. According to user needs, a standard Tessellation language (STL) file of the skeleton can be generated, or the skull boundary coordinates can also be exported. This file can be open by every computer-aided design (CAD) software, where the Solid Edge was used to create a 3D representation of the surface of the implant.

Although the most primitive way of implant design would be the vertical mirroring of the skull, it is sometimes too asymmetric, significantly damaged. There must be a smooth transition between the implant and the skull to avoid a conspicuous bump on the head of the patient. Our algorithm finds the outer boundary pixels of the skeleton on each layer and stores the coordinates of two points, where the line ends and two coordinates from the mirrored skull in the defective part. In the case of a dented skull, the continuity of curvature is scanned, and where the deviation reaches a limit, there is a dent. So, if the damage is on the right side of the head, first, we take the points with the highest value of $x$ coordinates. These boundary points are stored from each layer as illustrated in (Fig. 2), so this specified contour guarantees the implant fits the edge of the defect well. Two additional auxiliary points (in the missing region) are also be given in the first and last damaged plane if the hole is too large there. These auxiliary points can be assigned manually or derived from the vertically mirrored image. In this case, the mirrored model ensures the shape of the implant to be symmetric. These auxiliary points are also important, where the skull has an $S$ shape close to the temple. (Fig. 3) shows the designed implant.

\subsection{CAD model}

After performing series of morphological, and the existing parts are exported from MATLAB, the triangular surface model can be imported into the 
CAD system. Of course, it is helpful to deal only with the planes in the close area of the defect, thus saving considerable computing capacity. We need to transform the geometry into a CAD model to be manageable in the CAD system, which can be done in a few steps. (Fig. 4) shows the CAD model of the skull. The figure clearly shows the defect where the implant should be designed. The traditional design method is highly labor-intensive, and in even simpler cases, it requires at least 50 hours. The disadvantage of the conventional approach is that any form of modification (e.g., a doctor's unique needs in design) can only be accomplished with great difficulty and a lot of work. The sections of each slice must be modified one by one so that cohesion between them is maintained $[17,18]$.

The obtained spatial model of the implant is a geometry covered with small triangle elements, which is suitable for production (milling and additive production). However, if we want to scale a piece for expected use, then this format is no longer adequate, or the usability is limited. For such a complex geometry, scaling can only be accomplished by numerical simulation, finite element analysis, which requires a CAD

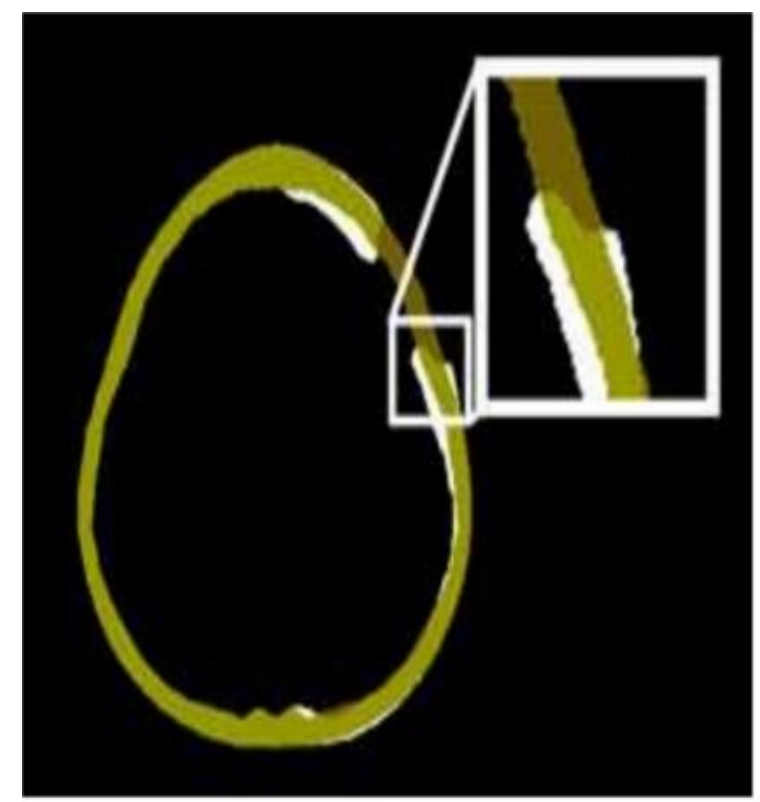

a geometry. Based on the individual CT images, the exact spatial coordinates of the missing parts in the given planes can be determined in the MATLAB software environment, which can be exported to a table. In the newer CAD systems, we can fit spatial CAD curves to the points scanned from the table. These are generally NURBS (Non-Uniform Rational Bezier Spline) curves, which offer the most modifications both globally and locally. In some cases (described earlier), we also need to give the bottom and top curves by auxiliary points. This defines the curvature in the plane of the sections. It is crucial that these ends of the curves join. These boundary curves can then be used to create a so-called boundary surface of which boundary curves are the curves we have defined. This has an interface that is continuously connected in tangent and curvature to the existing skull part. In many cases, the human skull has uneven, continuous curvature radius transition and may need to be modified in some regions. Adding any plane or planes to this and cutting our surface by them, we can create an interpenetration curve on the original surface too. (Fig. 5) shows the upper and lower limit curves in addition to the boundary Surface.

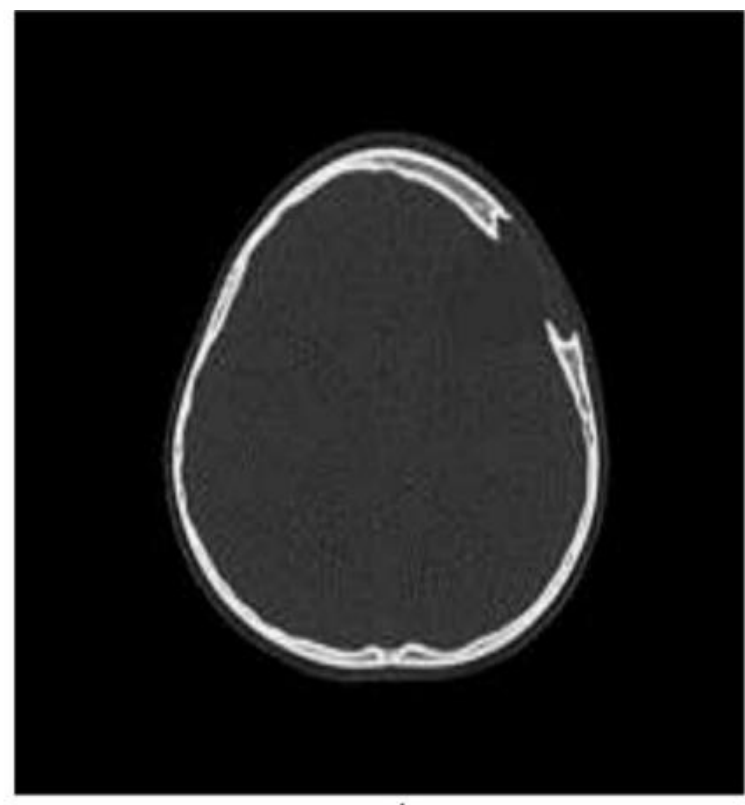

b

Fig. 1. Masking process. a Polygon shape around the damaged skull. b Damage skull after masking 

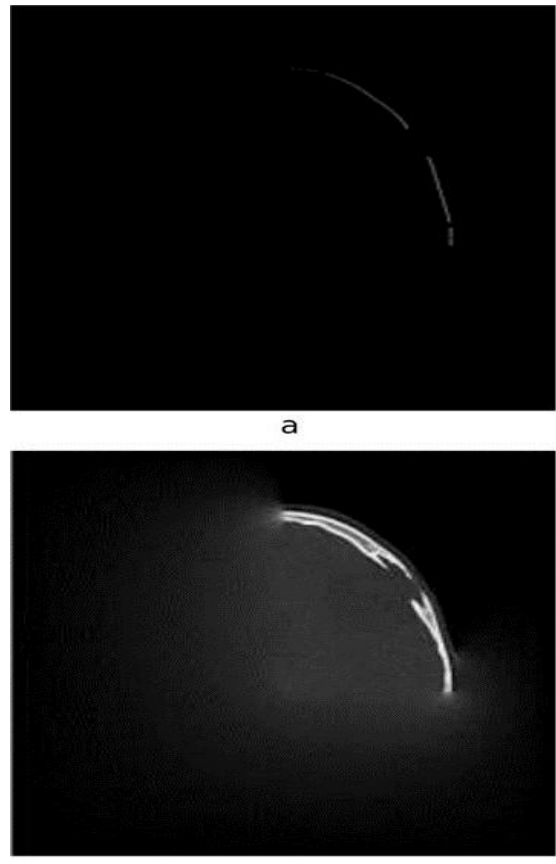
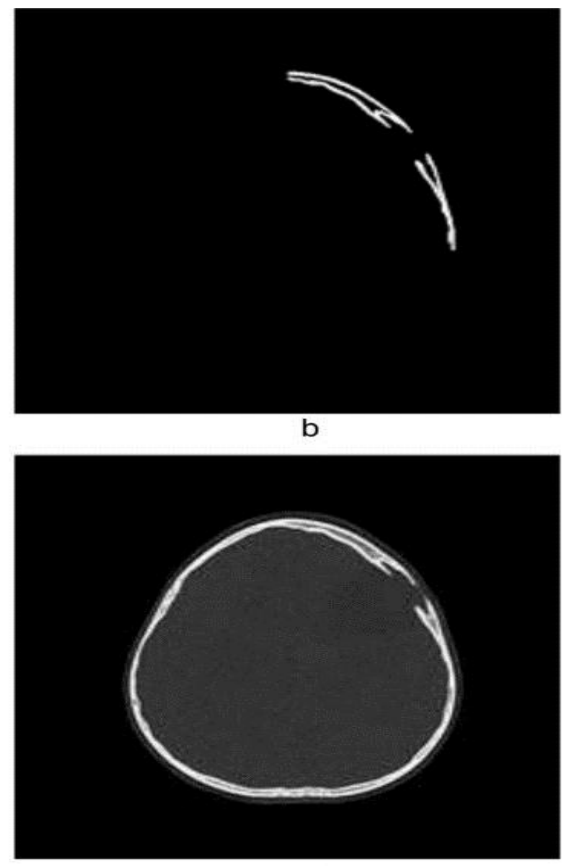

d

Fig. 2. Boundary points on each layer. a Layer 1. b Layer 2. c Layer 3. d Implant ready to fit the defected skull

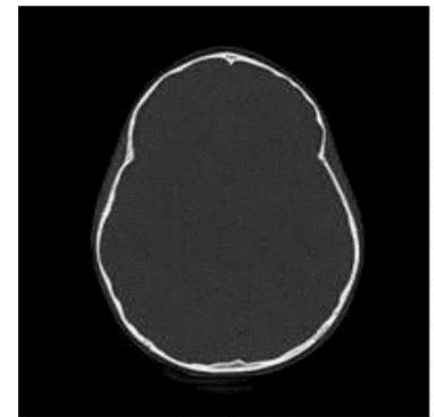

a

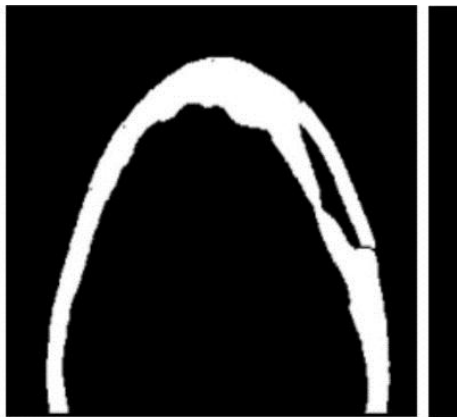

b

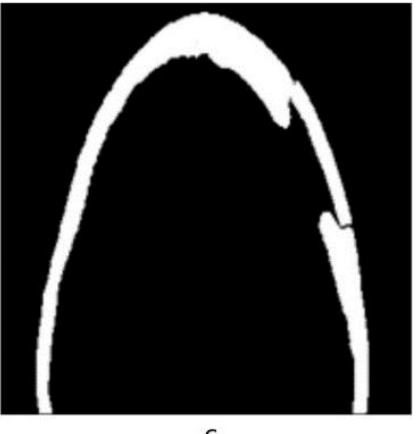

c

Fig. 3. Designed implant. a Mirrored image of the skull. b Implant and the two auxiliary points. c Skull implant

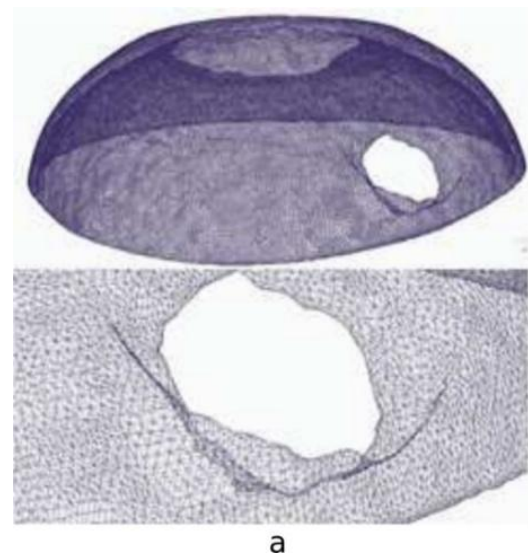

a

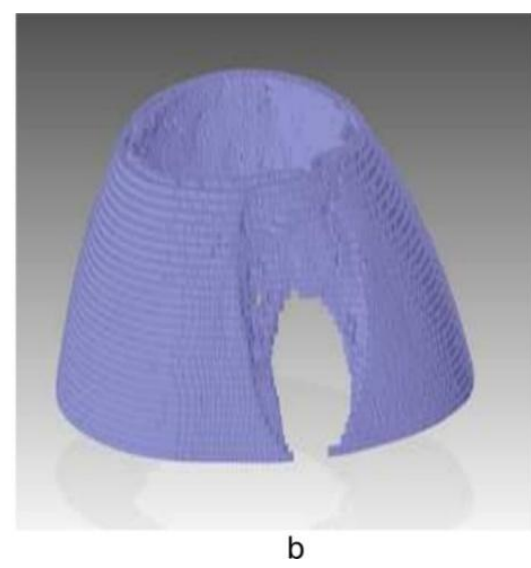

b

Fig. 4. CAD model. a Triangular surface model. b CAD model of the skull 


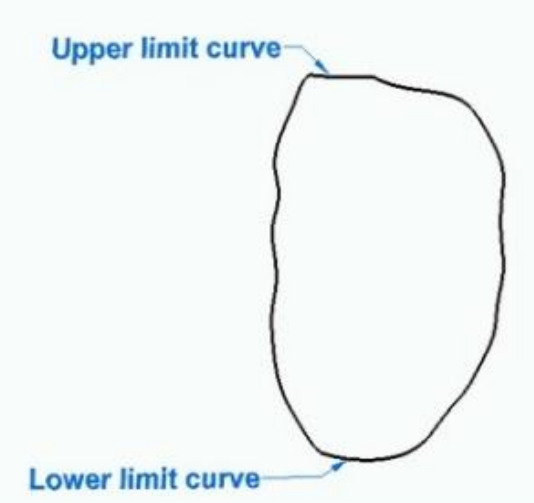

a

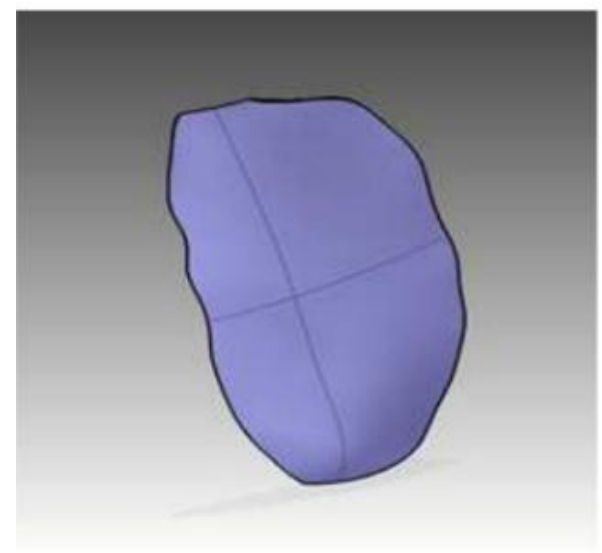

C

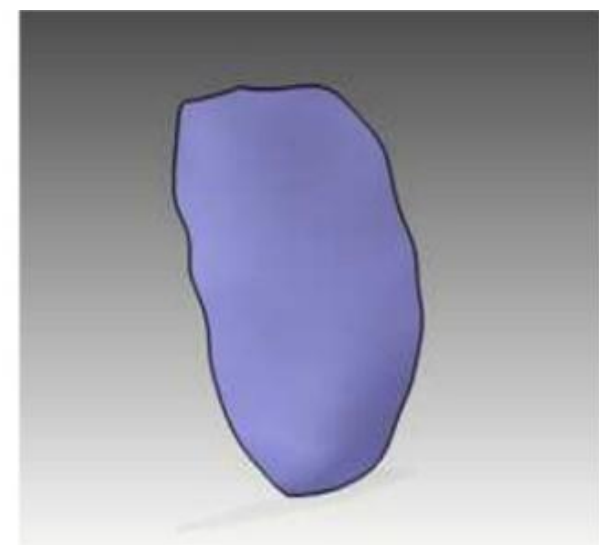

b

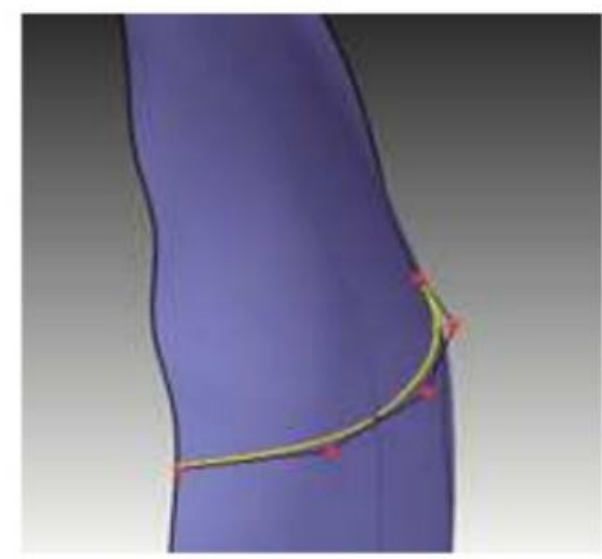

d

Fig. 5. Boundary Curves and surface. a Upper and lower limit curves. b Boundary surface. $c$ Interpenetration curve on the boundary surface. d NURBS curves

In fact, these curves are also NURBS curves that can be used to modify surface points. Of course, the degree of these curves can be increased arbitrarily ( $\mathrm{n}$-order, defined by $\mathrm{n}+1$ control points), so local protrusions, convex and concave portions can be implemented arbitrarily. So, we get a NURBS surface. This surface is associated with a given thickness (the expected thickness of the implant), and by defining the direction of the assignment, we can create a solid body model that perfectly matches the skull boundary surface. This can be done simply by chamfering. Concerning the concave surface depth, it is given by the doctor, the thickness of the implant is about half $(2 / 3)$ of the thickness of the skull. The edges of the skull are usually uneven in depth which must be straightened (conical shape) before implanting. This ensures the fit of the implant in depth. An essential need for the implant is that it has a conical shape, so it should be supported by the existing skull parts. Of course, the adequacy of the implant can only be verified in the installation environment. The implant geometry can be modified here as well. The implant design, according to the described method, can be accomplished in less than 1 hour as compared to the earlier 50 hours of labor demand while offering several modifications, and the handling of the model is much better. However, suppose any change is needed to the cranial model. In that case, it can be done quickly since our algorithm finds the outer boundary pixels of the skeleton on each layer and stores the coordinates of two points where the line ends. These two points from each layer are the contour of the outer layer of the implant. In case of a bigger hole in the skull, the user can give additional points manually (or using the mirrored skull) in the missing region of the skull to specify the shape of the implant. With these additional points, the form of the implant can be modified. The other way is to change it in CAD program using NURBS with different curvatures. (Fig. 6) shows the implant created using solidedge. 


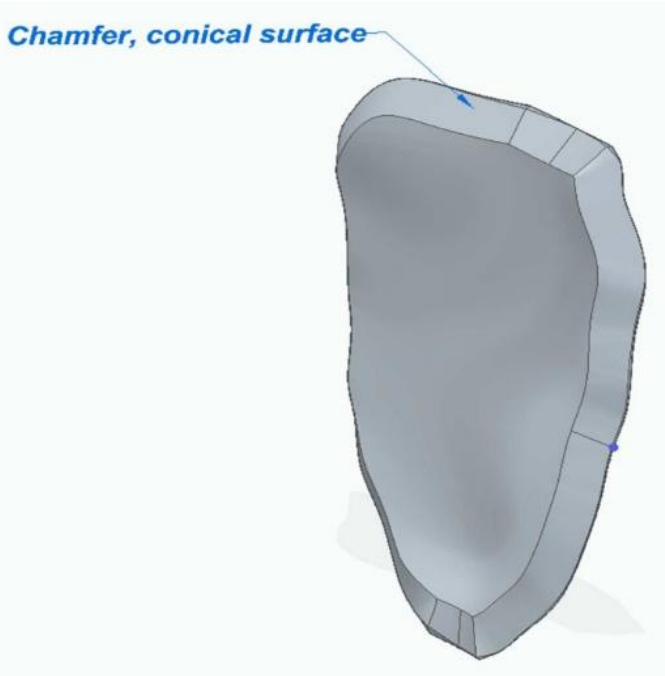

a

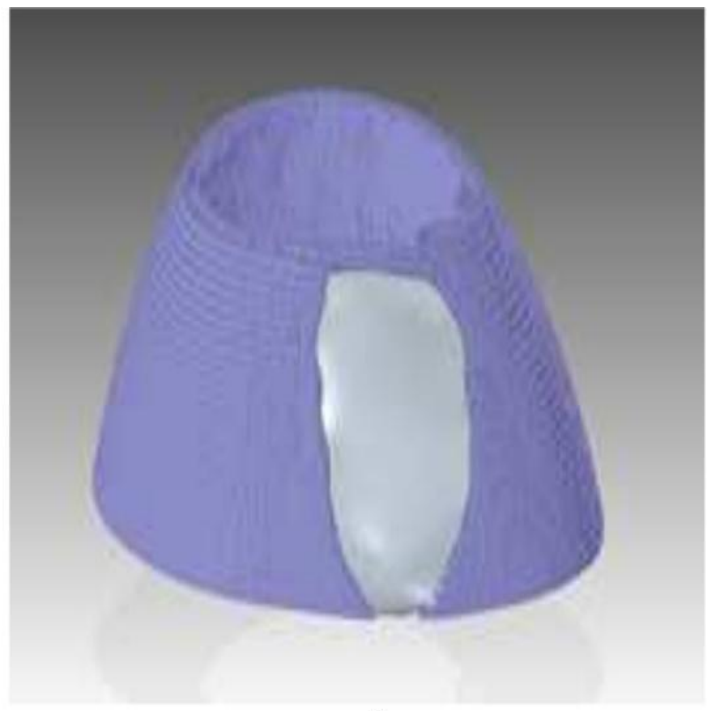

b

Fig. 6. Created implant. a Chamfer and conical shape. b Implant geometry

\section{RESULTS AND DISCUSSION}

Realistic 3D imaging and modeling are instrumental in the diagnosis and thus utilized in computer-aided surgery. Such a 3D model can be generated from the images obtained from computed tomography or magnetic resonance. In this process, the rendering of $3 \mathrm{D}$ anatomical models is based on the creation of a polygonal mesh that represents the geometrical properties of the object. With the utilization of medical image processing, CAD, and reverse engineering techniques, the new and simpler method for the determination of skull implant is presented in this study. Our computer-generated models can be exported as an STL file, which can further be used in rapid prototyping processes like 3D printing. The models are built layer-by-layer according to $3 \mathrm{D}$ datasets. The CAD skull implants have a huge advantage because their shape can be easily customized for the patient's requirements. These customized implants can even be used to replace large parts of the damaged skull and filled the gaps correctly. These implants are light in weight and have screw holes for fixing, making them very convenient to operate compared to conventional implants, which remain robust and wellintegrated with the skull even after a long period of time. The titanium alloy (Ti6Al4V) is the most commonly used material for manufacturing these implants with the help of additive manufacturing since it has high strength, high corrosion resistance, excellent biocompatibility, good osseointegration, low thermal expansion, and low thermal conductivity. Although additive manufacturing gained massive interest in medical applications, there is a limitation for this technology. One of the main drawbacks is the anisotropic mechanical behavior the manufactured part experienced. Thus, choosing the best-optimized printing parameters will positively affect the mechanical properties of the manufactured skull implant. The steps of the developed implant design process are shown in (Table 1), which can be found in the appendix.

An optional verification loop can be added to the algorithm if the implant shape is too complex, or the edge of the skull must be modified.

\section{CONCLUSION}

The skull is a protective cover of the brain, the sense organs, and the basis of the face. The defect in the skull reduces the protection of the brain. However, it causes psychological stress and social barriers for patients. Cranioplasty is a standard method used to repair the integrity of the cranial activity. In this paper, a new method of prosthesis modeling was presented. A novel algorithm was developed using MATLAB to build a model of a broken skull bone. The CT scan data was used to construct the STL 3D model. The Solidedge software was used to convert the STL 3D model into a CAD model. The implant can be designed by generating curve fitting, creating NURBS surface, adding thickness, and Chamfering. On the other hand, the 3D physical implant skull models can be manufactured using additive manufacturing. However, different 3D printers should be tested to maintain accuracy 
and feasibility. For the first time, our algorithm can identify the outer layers of the skull implant quickly and efficiently with less time-consuming. However, any modifications can be done quickly based on the human doctor's recommendation. Based on this methodology, automatic implant design and manufacturing processes can be implemented. Therefore, the novelty of this work builds a solid base for researchers to work on cranial implant designs. Additionally, a numerical analysis using finite elements can be carried out to obtain the maximum strength of different manufacturing materials. Eventually, the proposed method can save time for the manufacture of implant for surgeons up to 50 times shorter than the traditional way. Subsequently, we believe that our method is a promising technique and a convenient strategy that can be used by medical professionals to reconstruct the skull bone defects for patients.

\section{CONSENT}

Research NOT involving human participants, their data or biological material.

\section{ETHICAL APPROVAL}

This is an observational study. No ethical approval is required. Authors disclose all relationships or interests that could have direct or potential influence or impart bias on the work.

\section{COMPETING INTERESTS}

Authors have declared that no competing interests exist.

\section{REFERENCES}

1. Shweta $P$, Anburajan M. Finite element analysis of the skull implant using ANSYS Software, ICECT 2011 - $20113^{\text {rd }}$ Int. Conf. Electron. Comput. Technol. 2011;3:420425.

DOI: 10.1109/ICECTECH.2011.5941784.

2. Hieu LC, et al. A cheap technical solution for cranioplasty treatments, Technol. Heal. Care. 2004;12(3):281-292.

DOI: $10.3233 /$ thc-2004-12307.

3. De La Peña A, De La Peña-Brambila J, Pérez-De La Torre J, Ochoa M, Gallardo GJ. Low-cost customized cranioplasty using a 3D digital printing model: A case report. 3D Print. Med. 2018;4:1.

DOI: 10.1186/s41205-018-0026-7.
4. Sena K, Piyasin S. Determination of Average Contour of Thais Skulls for Design of Implants, Am. J. Eng. Appl. Sci. 2008;1(3):168-173.

DOI: 10.3844 /ajeassp.2008.168.173.

5. Eufinger $H$, Wehmöller $M$, Harders $A$, Heuser L. Prefabricated prostheses for the reconstruction of skull defects, Int. J. Oral Maxillofac. Surg. 1995; 24(1)Part 2:104110.

DOI: 10.1016/S0901-5027(05)80870-7.

6. Chrzan R, Urbanik A, Karbowski K, Moskała M, Polak J, Pyrich M. Cranioplasty prosthesis manufacturing based on reverse engineering technology. Med. Sci. Monit. 2012;18(1):1-6.

DOI: $10.12659 / \mathrm{msm} .882186$.

7. Salyer KE, Taylor DP. Bone grafts in craniofacial surgery. Clin. Plast. Surg. 1987;14(1):27-35.

DOI: $10.1055 / \mathrm{s}-0029-1215875$.

8. de S. Leão R, et al. Complications with PMMA compared with other materials used in cranioplasty: A systematic review and meta-analysis. Braz. Oral Res. 2018;32: e31.

DOI:10.1590/1807-3107bor-

2018.vol32.0031.

9. D'Urso F, Effeney PS, Earwaker DJ, Barker WJ, Redmond TM, Thompson MJ, Tomlinson RG. Custom cranioplasty using stereolithography and acrylic. British Journal of Plastic Surgery. Br. J. Plast. Surg. 2000;53(3):200-204.

DOI: $10.1054 /$ bjps.1999.3268.

10. Sailer HF, Haers PE, Zollikofer CPE, Warnke T, Carls FR, Stucki P. The value of stereolithographic models for preoperative diagnosis of craniofacial deformities and planning of surgical corrections. Int. J. Oral Maxillofac. Surg. 1998;27(5):327-333.

DOI: 10.1016/S0901-5027(98)80059-3.

11. Bill JS, et al. Stereolithography in oral and maxillofacial operation planning. Int. J. Oral Maxillofac. Surg. 1995;24(1)Part 2:98-103. DOI: 10.1016/S0901-5027(05)80869-0.

12. Jardini $A L$, et al. Cranial reconstruction: $3 D$ biomodel and custom-built implant created using additive manufacturing. J. CranioMaxillofacial Surg. 2014;42(8):18771884.

DOI: 10.1016/j.jcms.2014.07.006.

13. Lin YC, Cheng CY, Cheng YW, Shih CT. Skull repair using active contour models Procedia Manuf. 2017;11:2164-2169. DOI: 10.1016/j.promfg.2017.07.362. 
14. Mustra M, Delac K, Grgic M. Overview of the DICOM standard. Proc. Elmar - Int. Symp. Electron. Mar. 2008;1: 39-44.

15. Ficzere $\mathrm{P}$, Bogya $\mathrm{P}$, Horváth $\mathrm{E}$, Lovas $\mathrm{L}$. Simplified cad model of human metacarpal for implantation péter ficzere-péter bogyaeszter horváth-lászló lovas. 2018; 8(2):512.

16. Winder J, Bibb R. Medical rapid prototyping technologies: State of the art and current limitations for application in oral and maxillofacial surgery. J. Oral Maxillofac. Surg. 2005;63(7):1006-1015. DOI: 10.1016/j.joms.2005.03.016.

17. Gyori M, Ficzere P. Increasing role of sections caused by 3D modelling. Period. Polytech. Transp. Eng. 2016;44(3):164171.

DOI: 10.3311/PPtr.9053.

18. Gyori M, Ficzere P. Use of sections in the engineering practice. Period. Polytech. Transp. Eng. 2017;45(1):21-24.

DOI: 10.3311/PPtr.9144. 


\section{APPENDIX}

Table 1. Steps of implant design process

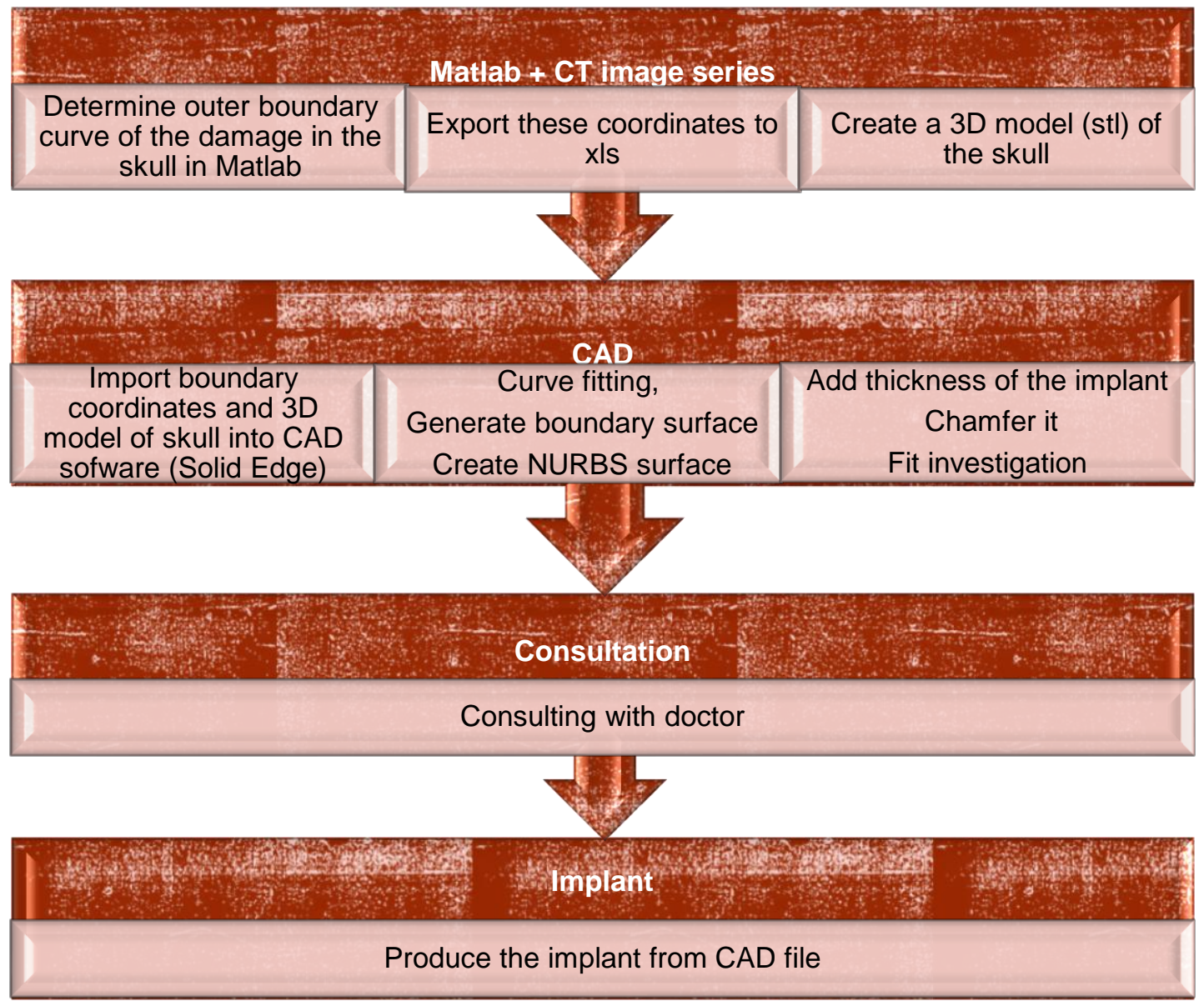

(C) 2021 Markiz et al.; This is an Open Access article distributed under the terms of the Creative Commons Attribution License (http://creativecommons.org/licenses/by/4.0), which permits unrestricted use, distribution, and reproduction in any medium, provided the original work is properly cited.

Peer-review history:

The peer review history for this paper can be accessed here: https://www.sdiarticle4.com/review-history/68649 